\title{
Catheter Ablation for Atrial Fibrillation: The Tailored Approach
}

\author{
Luigi Sciarra ${ }^{1}$, Zefferino Palamà ${ }^{2 *}$ and Martina Nesti ${ }^{3}$ \\ ${ }^{1}$ Cardiology Unit, Policlinico Casilino, Italy \\ ${ }^{2}$ Cardiology Unit, Ospedale "SS. Annunziata", Italy \\ ${ }^{3}$ Cardiology Unit - Cardiovascular and Neurological Department, Italy
}

*Corresponding author: Zefferino Palamà, SC Cardiologia, Ospedale “SS Annunziata”, via F. Bruno, 1 74100, Taranto, Italy

\begin{tabular}{|c|c|}
\hline ARTICLE INFO & ABSTRACT \\
\hline Received: 幽 June 10, 2019 & Abbreviations: AF: Atrial Fibrillation; PV: Pulmonary Veins; CFAE: Complex Fragmented \\
\hline Published: 豐 June 17, 2019 & Atrial Electrograms \\
\hline
\end{tabular}

Citation: Boshra I Arnout, Oleg Y Latyshev, Ahed J Alkhatib. Catheter Ablation for Atrial Fibrillation: The Tailored Approach. Biomed J Sci \& Tech Res 18(5)2019. BJSTR. MS.ID.003217.

\begin{abstract}
Mechanisms of Atrial Fibrillation: Brief Historical Notes
The study of atrial fibrillation (AF) pathophysiology represents a challenge that is not yet completely understood. The first studies date back to the early twentieth century, but the fundamental steps that have led to current knowledge on this arrhythmia have developed in the last 25 years. In 1924 Garrey [1] published a review on the "auricular fibrillation" indicating the mechanisms considered responsible for AF. The author proposed three main models in which this arrhythmia was sustained: multiple or single ectopic foci with fibrillation conduction, a single re-entry circuit (defined mother wave) with fibrillation conduction and multiple simultaneous re-entry circuits [1]. Between the end of the 60 s and the early 70s, Coumel postulated the concept of the triangle needed to support every type of arrhythmia: triggers, arrhythmogenic substrate and modulating factors. In this model, although historically dated, atrial fibrillation still fits adequately. These mechanisms have been proposed to play a crucial role in the $\mathrm{AF}$ genesis and every part of the triangle may coexist in the same patients [2].
\end{abstract}

Trigger: Pulmonary Veins and Non-Pulmonary Veins Triggers

The real turning point in the long history of the AF was in 1998 when Haissaguerre first demonstrated the role of pulmonary veins (PV), offering a still valid target for a pathophysiological approach to AF ablation, through the electrical isolation of them [3]. The anatomical, histological and electrical discontinuity in the area between PVs and the left atrium has provided basis for several studies aimed at identifying the pathophysiological mechanisms responsible for the $\mathrm{AF}$ initiation and maintenance. The atrial myocardium consists of one to three muscular layers superimposed according to the regions and extends for $1-3 \mathrm{~cm}$ to cover the initial PVs tracts, forming muscular sleeves that surround veno-atrial connecting zone [4]. These sleeves play a role in the regulation of pulmonary venous flow. Both for their histological properties and for their cellular peculiarities, the sleeve's myocytes represent an important arrhythmogenic substrate. From a histological point of view, this zone of veno-atrial connection represents an architectural 
discontinuity point; this discontinuity promotes micro-reentry mechanisms and constitutes a substrate that can trigger rapid focal ectopic activity. This early ectopic activity, originating from PVs hosts, manifests itself with the so-called electrocardiographic "P on T" phenomenon, with very early extra systolic beats, as an expression of the short refractory nature of the pulmonary vein muscles (Figure 1). Focal extrapulmonary foci, which can trigger atrial fibrillation, such as the Marshall ligament, the crista terminalis, the coronary sinus ostium, the posterior wall of the left atrium or more commonly the superior vena cava are described in literature, as well [5] (Figure 1).

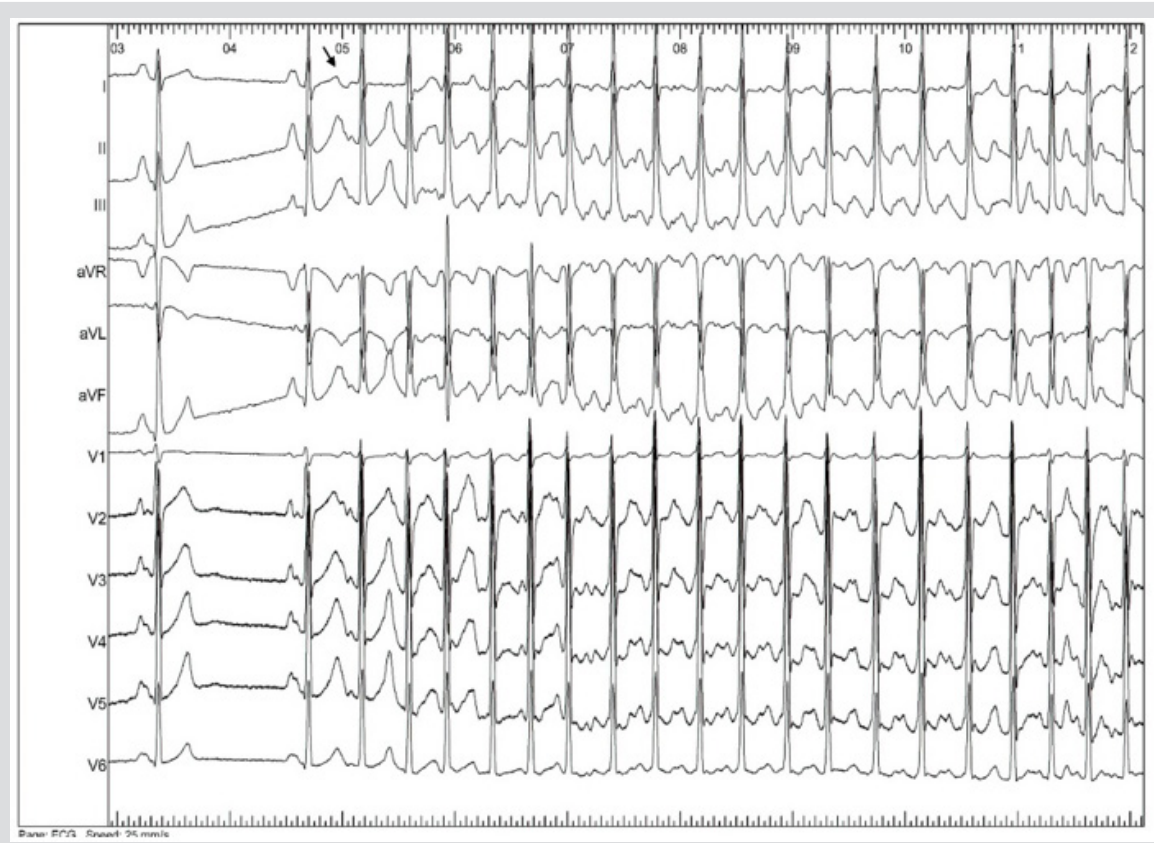

Figure 1: "P on T" phenomenon on surface ECG. After the first two sinus beats, a very early atrial ectopic premature beat (see the arrow) triggers atrial fibrillation. The ectopic beat is so premature that the $\mathrm{P}$ wave is inscribed inside the $\mathrm{T}$ wave of the previous beat ( $\mathrm{P}$ on $\mathrm{T}$ phenomenon). This behaviour is typical for pulmonary veins ectopic beats triggering atrial fibrillation.

\section{Substrate}

Particularly in patients with persistent $\mathrm{AF}$, rather than in paroxysmal, the role of the atrial substrate is considered to be crucial. The substrate for AF maintenance is a complex concept and includes all the atrial myocardium structural and functional abnormalities that can sustain AF. There are numerous attempts to approach the substrate modification in the contest of AF ablation. In 2004, Nademanee group identified complex fragmented atrial electrograms (CFAE) capable of generating micro-reentry phenomena as possible critical areas for the AF substrate [6]. Other approaches such as linear lesions (roof, mitral isthmus, anterior line) have been described for substrate modification [7]. However, conclusive data supporting efficacy of extra pulmonary vein ablative approaches for atrial substrate modification are still lacking (STAR AF study) [8].

\section{Modulating Factors: The Role of Autonomic Nervous System}

The autonomic nervous system exerts important electrophysiological effects that can favor arrhythmic phenomena development, including atrial fibrillation. Supporting this concept, it has been demonstrated a circadian rhythm of some episodes of paroxysmal AF [9], and this can be related to different parasympathetic or sympathetic drives. A parasympathetic dominance shortens the effective refractory period of atrial tissue and increases automaticity and triggered activity. Ganglionated plexi ablation demonstrated to be effective in some paroxysmal $\mathrm{AF}$ patients' groups [10]. Even the adrenergic tone appears to be involved in $\mathrm{AF}$ initiation and/or maintenance. Catecholamines may produce membrane potential oscillations characteristic of after-depolarisations, by increasing $\mathrm{Ca} 2+$ current, $[\mathrm{Ca} 2+] \mathrm{i}$ and consequent $\mathrm{Na}+-\mathrm{Ca} 2+$ exchange and may also enhance automaticity. Indeed, the suppression of $\mathrm{AF}$ by $\beta$-blocker treatment may involve an attenuation of arrhythmic activity that is caused by increased [Ca2+]i, coupled with effects of adaptation to the treatment [11].

\section{Supporting a Tailored Approach to Ablate AF}

Why to sustain a tailored approach for AF ablation today? The first answer could be: because the AF ablation results are good and encouraging but still far from $100 \%$. The Fire and ICE study [12] have been developed to demonstrate the non-inferiority of the cryo-ablation versus radiofrequency ablation of pulmonary veins, in patients with paroxysmal AF. The study endpoint was reached, but with both techniques the success rates in terms of freedom from $\mathrm{AF}$ recurrences did not overcome the 70 percent at 1 year and a half follow-up [12]. Many doctors and cardiologists feel that those results resemble more properly the real-life data, and some skepticism about AF ablation real efficacy is not uncommon. 
Moreover, the price that we pay in terms of complications related to AF ablation is still remarkable in real life [13]. Some ablative complications, even if very uncommon, are to be considered serious and hardly acceptable in a view of a treatment of an arrhythmia generally considered as a benign one. An important answer, in order to improve AF ablation efficacy and to reduce complications is surely technological progresses. New ablative materials, new diagnostic catheters, new mapping systems, new software's, new systems that allow contact-force monitoring of the ablative catheters and many other tools are everyday available: in the electrophysiological lab today we can be assisted by a technological support that we cannot ever imagine twenty years ago [14-17].

Technological issue is very important but cannot be the only answer. Because the AF pathogenesis is multifactorial and complex, a standardized and primarily anatomic ablation strategy may not be sufficient to eliminate all the arrhythmogenic mechanisms. To date, too often patients are approached with a first line ablative strategy without a proper clinical or electrophysiological classification. We believe that in many cases it is possible to tailor the ablative strategy on the arrhythmia mechanism of a given patients, thus improving safety and efficacy of AF ablation. In 2011 we demonstrated that a significant proportion $(10.1 \%)$ of candidates to $\mathrm{AF}$ ablation are inducible for an underlying supraventricular tachycardia, and its ablation showed a preventive effect on AF recurrences, with very high success rate [18]. We know that this is not possible in every patient, but this does not mean that is not worth trying to reconstruct the precise arrhythmogenic mechanism in the single patients. Some patients may have extra pulmonary veins ectopic foci $[19,20]$ : in such a populations pulmonary veins isolation may not be the appropriate therapy.

Some other patients clearly show a single arrhythmogenic pulmonary vein that sometimes can be suspected only by properly analyzing the surface ECGs, capturing AF beginning [21]. An ablative approach tailored only on the culprit vein isolation could be safe and effecting, avoiding unuseful lesions. As already mentioned, selected patients may have a prevalent vagal form of arrhythmia and in those cases parasympathetic ganglia ablation may be a reasonable solution [10]. How to re-construct the electrophysiological mechanism of AF and to tailor the ablative approach? To be honest this issue is not a simple one, and, as already said, not always possible. But we cannot understand why AF has to be considered differently from any other disease. In medicine we know that the correct sequence is: first the diagnosis and the pathophysiology and then the therapy! First of all we like to underline the importance of simple tools as, for instance, the patients' interview. This is a low-cost procedure but can be very useful. Does the arrhythmic palpitations are preceded by regular palpitations? Do the arrhythmia is preceded by a physical effort, or occurs during exercise?

Does the arrhythmia start after big meals or by night while asleep? If so, we could be oriented to a vagal-mediated AF, that could benefit from ganglionated plexi ablation. Moreover, it is necessary to consider all the available tools that can help to identify the mechanisms that trigger or sustain AF. To date invasive and non-invasive ECG monitoring systems, involving Holter monitoring, external event recorders and/or loop recorders, implantable loop recorders may significantly help to identify AF triggers or mechanisms in general. When we don't have enough clinical information from the noninvasive tests, we can perform an electrophysiological study before ablation, in order to investigate on non-pulmonary veins triggers. This procedure is not so time consuming and has been demonstrated to help in selecting subgroup of patients that may benefit of limited and very effective ablative approach [18]. There are possible pitfalls of the tailored ablative strategy. The most important one is that it can be timeconsuming thus preventing an early ablative approach. It is well known the concept "atrial fibrillation begets atrial fibrillation" [22]: a delayed ablation could favor the progression of the arrhythmia.

This is true, but it has been demonstrated that only a minority of patients with paroxysmal AF evolves towards persistent forms and this is particularly true in young patients with "lone AF". The rate of progression can however be very different in different patients. Sometimes AF presents itself as a persistent form, sometimes it tends to keep paroxysmal behavior and in any case this behavior is strongly influenced by co-morbidities (hypertension, diabetes, obesity) [23]. We totally agree with scientists sustaining that tailoring the ablative strategy on $\mathrm{AF}$ is not always possible, but we do not think that this is a good reason not to try. Atrial fibrillation represents a complex, multifactorial arrhythmia. A correct clinical and electrophysiological classification of patients is crucial to improve the success rates of our treatments.

\section{References}

1. Garrey WE (1924) Auricular fibrillation. Physiol Rev 4: 215-250.

2. Coumel P, Leenhardt A (1993) Mental Cardiac arrhytmias and the autonomic nervous system. J Cardiovasc Electrophysiol 4: 338-355.

3. Haïssaguerre M, Jaïs P, Shah DC, Takahashi A, Hocini M, et al. (1998) Initiation of atrial fibrillation by ectopic beats originating in the pulmonary veins. N Engl J Med 339(10): 659-666.

4. Ho SY, Cabrera JA, Tran VH, J Farre, R Anderson, et al. (2001) Architecture of the pulmonary veins: relevance to radiofrequency ablation. Heart 86(3): 265-70.

5. Lin WS, Tai CT, Hsieh MH, Tsai CF, Lin YK, et al. (2003) Catheter ablation of paroxysmal atrial fibrillation initiated by non-pulmonary vein ectopy. Circulation 107(25): 3176-3183.

6. Nademanee K, Mc Kenzie J, Kosar E, Schwab M, Sunsaneewitayakul B, et al. (2004) A new approach for catheter ablation of atrial fibrillation: mapping of the electrophysiologic substrate. J Am Coll Cardiol 43(11): 2044-2053.

7. Kottkamp H, Tanner H, Kobza R, Schirdewahn P, Dorszewski A, et al. (2004) Time courses and quantitative analysis of atrial fibrillation episode number and duration after circular plus linear left atrial lesions: trigger elimination or substrate modification: early or delayed cure? J Am Coll Cardiol 44(4): 869-877.

8. Verma A, Mantovan R, Macle L, De Martino G, Chen J, et al. (2010) Substrate and Trigger Ablation for Reduction of Atrial Fibrillation (STAR 
AF): a randomized, multicentre, international trial. Eur Heart J 31(11): 1344-1356.

9. Leiria TL, Glavinovic T, Armour JA, Cardinal R, De Lima GG, et al. (2011) Longterm effects of cardiac mediastinal nerve cryoablation on neural in- ducibility of atrial fibrillation in canines. Auton Neurosci 161(1-2): 68-74.

10. Calò L, Rebecchi M, Sciarra L, De Luca L, Fagagnini A, et al. (2012) Catheter ablation of right atrial ganglionated plexi in patients with vaga paroxysmal atrial fibrillation. Circ Arrhythm Electrophysiol 5(1): 22-31.

11. Workman A J (2010) Cardiac adrenergic control and atrial fibrillation. Naunyn Schmiedebergs Arch Pharmacol 381(3): 235-249.

12. Kuck KH, Brugada J, Fürnkranz A, Metzner A, Ouyang F, et al. (2016) Cryoballoon or Radiofrequency Ablation for Paroxysmal Atrial Fibrillation. N Engl J Med 374(23): 2235-2224.

13. Cappato R, Calkins H, Chen SA, Davies W, Iesaka Y, et al. (2010) Updated worldwide survey on the methods, efficacy, and safety of catheter ablation for human atrial fibrillation. Circ Arrhythm Electrophysiol 3(1): 32-38.

14. Sciarra L, Golia P, Natalizia A, De Ruvo E, Dottori S, et al. (2014) Which is the best catheter to perform atrial fibrillation ablation? A comparison between standard ThermoCool, SmartTouch, and Surround Flow catheters. J Interv Card Electrophysiol 39(3): 193-200.

15. Hussein A, Das M, Chaturvedi V, Asfour IK, Daryanani N, et al. (2017) Prospective use of Ablation Index targets improves clinical outcomes following ablation for atrial fibrillation. J Cardiovasc Electrophysiol 28(9): 1037-1047.

\section{ISSN: 2574-1241}

DOI: 10.26717/BJSTR.2019.18.003217

Zefferino Palamà. Biomed J Sci \& Tech Res

This work is licensed under Creative Commons Attribution 4.0 License

Submission Link: https://biomedres.us/submit-manuscript.php
16. Sciarra L, Iacopino S, Palamà Z, De Ruvo E, Filannino, et al. (2018) Impact of the third generation cryoballoon on atrial fibrillation ablation: An useful tool? Indian Pacing Electrophysiol J 18(4): 127-132.

17. Scarà A, Sciarra L, De Ruvo E, Borrelli A, Grieco D, et al. (2018) Safety and feasibility of atrial fibrillation ablation using Amigo system versus manual approach: A pilot study. Indian Pacing Electrophysiol J 18(2): 6167.

18. Sciarra L, Rebecchi M, De Ruvo E, De Luca L, Zuccaro LM, et al. (2010) How many atrial fibrillation ablation candidates have an underlying supraventricular tachycardia previously unknown? Efficacy of isolated triggering arrhythmia ablation. Europace 12(12): 1707-1712.

19. Higa S, Tai CT, Chen SA (2006) Catheter ablation of atrial fibrillation originating from extrapulmonary vein areas: Taipei approach. Heart Rhythm 3(11): 1386-1390.

20. Santangeli P, Zado ES, Hutchinson MD, Riley MP, Lin D, et al. (2016) Prevalence and distribution of focal triggers in persistent and longstanding persistent atrial fibrillation. Heart Rhythm 13(2): 374-382.

21. Sciarra L, Corò L, Bocchino M, Sitta N, Marras E, et al. (2004) Ablation of a single pulmonary vein arrhythmic focus triggering several supraventricular arrhythmias. Ital Heart J 5(12): 946-950.

22. Wijffels MC, Kirchhof CJ, Dorland R, Maurits A Allessie (1995) Atrial fibrillation begets atrial fibrillation. A study in awake chronically instrumented goats. Circulation 92(7): 1954-1968.

23. Jahangir A, Lee V, Friedman PA, Trusty JM, Hodge DO, et al. (2007) Longterm progression and outcomes with aging in patients with lone atrial fibrillation: a 30-year follow-up study. Circulation 115(24): 3050-3056.

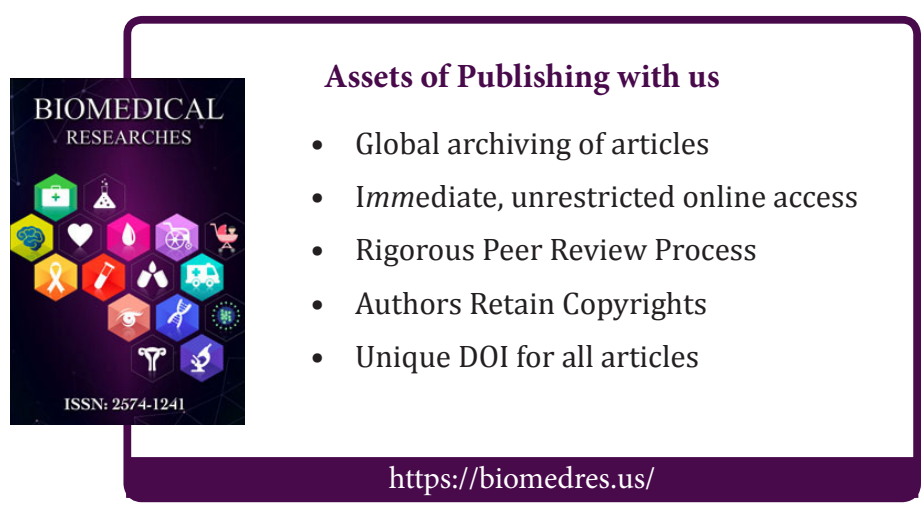

